Musées, Patrimoine et Culture scientifiques et techniques

$164 \mid 2016$

mars-avril 2016

\title{
Atome Hôtel : un web-documentaire pour revisiter le tableau périodique des éléments
}

Thierry Brassac et Agnès Pesenti

\section{OpenEdition \\ Journals}

Édition électronique

URL : http://journals.openedition.org/ocim/1647

DOI : $10.4000 /$ ocim. 1647

ISSN : 2108-646X

Éditeur

OCIM

Édition imprimée

Date de publication : 1 mars 2016

Pagination : 28-32

ISSN : 0994-1908

Référence électronique

Thierry Brassac et Agnès Pesenti, « Atome Hôtel : un web-documentaire pour revisiter le tableau périodique des éléments », La Lettre de l'OCIM [En ligne], 164 | 2016, mis en ligne le 01 mars 2017, consulté le 02 mai 2019. URL : http://journals.openedition.org/ocim/1647 ; DOI : 10.4000/ocim.1647

Ce document a été généré automatiquement le 2 mai 2019.

Tous droits réservés 


\section{Atome Hôtel : un web-documentaire pour revisiter le tableau périodique des éléments}

\section{Thierry Brassac et Agnès Pesenti}

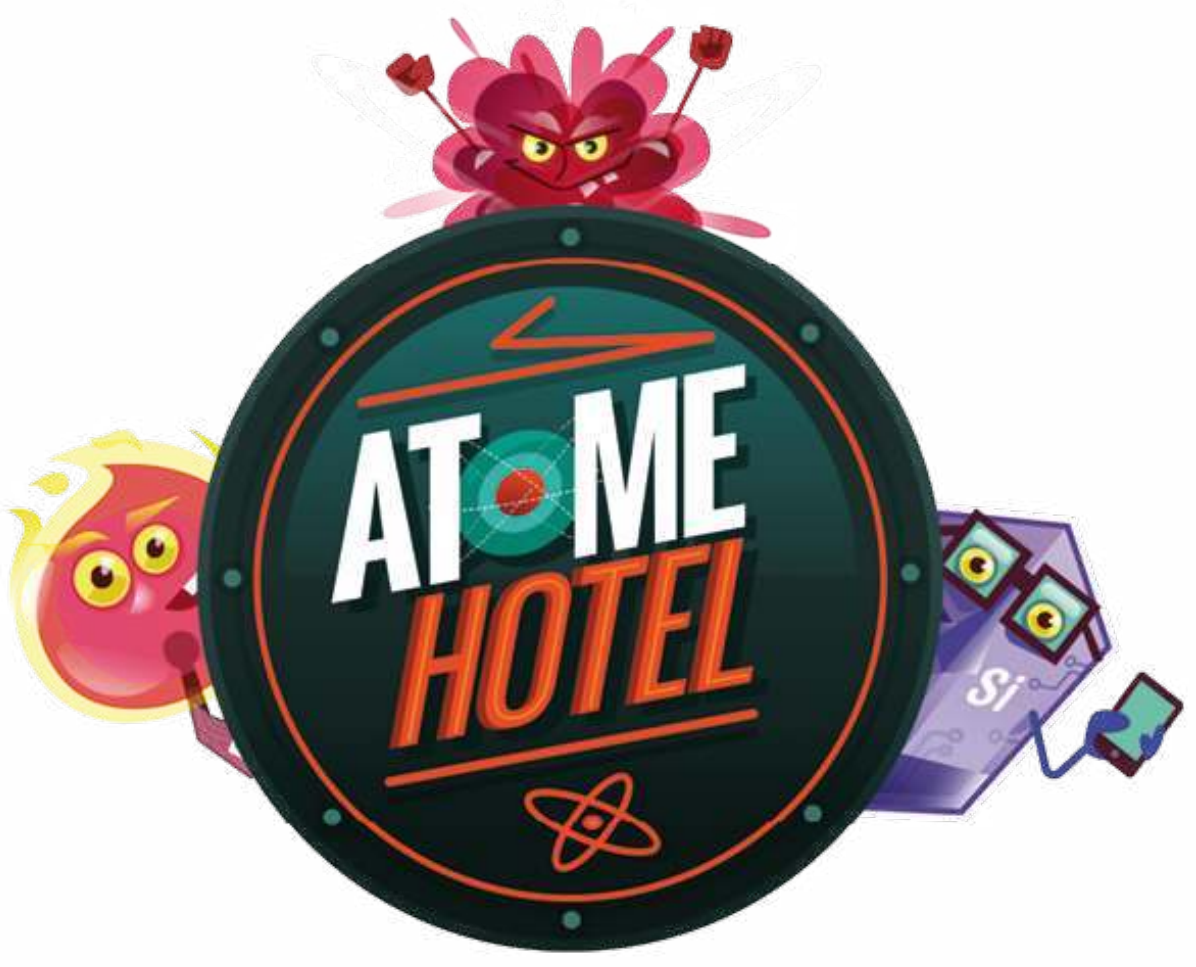

(c) Loguy-Bot 42

www.atome-hotel.fr est un outil numérique qui permet d'aborder le monde de la chimie en partant à la rencontre des atomes du tableau périodique. En le visitant, on y découvre 
pourquoi les rubis sont rouges, depuis quand l'or est utilisé comme monnaie, quelle est la valeur d'un diamant ou pourquoi les physiciens adorent l'atome d'hélium...

Imaginez un hôtel virtuel et numérique dédié au monde des atomes. Il contient 114 chambres destinées à accueillir les 114 atomes du tableau périodique des éléments. À la réception, Dmitri, la mascotte officielle, invite à une visite des "suites atomiques" occupées par une quarantaine d'atomes ludiques, colorés et animés. Tous sont personnifiés et certains, comme l'hydrogène, l'or, le radium ou le lithium, sont féminisés.

La saison 1 d'Atome Hôtel propose 25 courts documentaires et des interviews dans lesquelles des chercheurs nous parlent de leur atome préféré. On y trouve également 5 road-trips au cours desquels Dmitri visite des laboratoires nationaux et internationaux travaillant sur les atomes. Cette première saison offre au total 120 minutes de films, une dizaine d'interviews, des chroniques sonores, des citations, quiz, jeux... ainsi que des images d'archives de l'INA.

4 Le tableau périodique des éléments, sous sa forme classique, est connu dans le monde entier. C'est un référentiel universel des salles de classe de physique-chimie qui peut laisser songeurs, faire rêver ou cauchemarder les collégiens et les lycéens. Également appelé table de Mendeleïev, il date de 1869 et a été créé par le célèbre chimiste russe Dmitri Ivanovitch Mendeleïev (1834-907). Il regroupe tous les éléments chimiques connus, classés en fonction de leurs propriétés physico-chimiques et caractéristiques. Depuis sa publication, il a connu de nombreux réajustements jusqu'à prendre la forme que nous lui connaissons aujourd'hui : il comptait 63 atomes à sa création, contre 114 aujourd'hui.

5 L'indispensable casse-tête mnémotechnique de la classification des éléments constitue toujours une ressource importante pour l'étude de la chimie au collège et au lycée. L'idée de le dépoussiérer et le revisiter est venue de Thierry Brassac, responsable du service de culture scientifique à l'université de Montpellier: "L'idée était de partir d'un décalage, de transformer un tableau austère avec plein de constantes physiques en un tableau hyper-ludique avec des personnages un peu foufous et plein d'humour; et de l'exploiter avec le plus grand sérieux ".

\section{Un projet de culture scientifique au long cours}

6 Le projet prend racine en 2011, proclamée "Année Internationale de la Chimie" par l'UNESCO. Dès lors, le service de culture scientifique de l'université de Montpellier a recherché ce qui pouvait faire sens pour le grand public et trouvé une icône indétrônable de la chimie : le Tableau Périodique des Éléments (TPE). Lorsqu'on prend garde de le mettre en perspective historique, ce tableau constitue rien de moins que l'essai et la réussite conceptuelle de proposer une classification de tous les atomes qui constituent notre Univers.

7 L'équipe culture scientifique de l'université de Montpellier a donc décidé de trouver les moyens de revisiter ce monument d'histoire des sciences afin de le rendre attractif et ludique.

8 Un travail préliminaire avec Gilles Macagno (professeur de Sciences de la Vie et de la Terre, illustrateur, éditeur et auteur de la revue Drosophile, www.drosophile.net) a consisté à personnifier les atomes sous forme de personnages de type bande dessinée. Létape suivante du projet a été de demander à des chercheurs quel était leur atome préféré. L'idée était de trouver un point d'entrée original permettant d'établir une 
discussion avec le scientifique à propos de son travail quotidien par le biais de l'affectif. C'est ainsi que des binômes atomes/chercheurs susceptibles de nous fournir de belles histoires de sciences à mettre en images ont été constitués. C'est une étape qui a donné lieu à des rencontres éclectiques et passionnantes !

Quant au héros du projet, il s'est imposé de lui-même en la personne de Dmitri Mendeleïev, le "papa" du tableau périodique. Son apparence stéréotypée de savant, son obsession pour le classement des atomes, ses intuitions et son génie en faisaient une mascotte idéale...

10 L'achèvement de la première saison a nécessité un peu plus de trois ans de travail collaboratif. Réalisée avec le plus grand sérieux, elle a réuni des chercheurs de l'université de Montpellier, des scientifiques internationaux et une équipe créative spécialisée dans le multimédia. La production déléguée a été confiée à Pages \& Images Productions, une société basée à Montpellier. Son rôle: rassembler les compétences, coordonner l'ensemble avec une dimension artistique et se charger des aspects financiers. Dès le début du partenariat, le service de culture scientifique de l'université de Montpellier a été séduit par leur philosophie d'écriture et leur approche particulière du documentaire via les nouveaux médias.

11 Au final, Atome Hôtel est un outil de vulgarisation scientifique, accessible dès l'âge de 12 ans, qui permet à un public diversifié et curieux d'aborder le monde des atomes de manière vivante et originale.

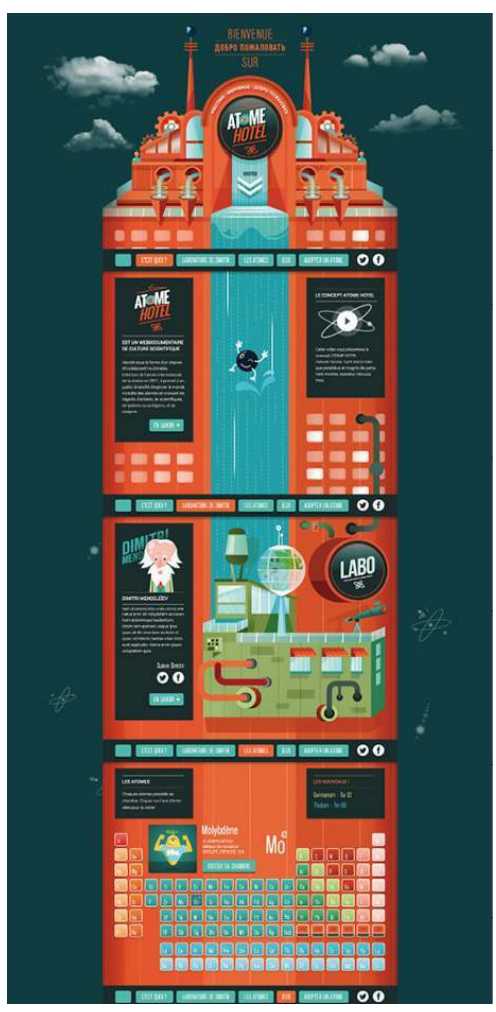

(c) Loguy-Bot 42

\section{L'aboutissement d'un travail collaboratif}

12 Atome Hôtel est une production Pages \& Images, en co-production avec l'université de Montpellier, Universcience et Francetv Éducation et en partenariat avec Le Réseau 
Canopé, l'Académie de Montpellier et l'INA. La diffusion est assurée par Francetv Éducation, Universcience et la Radio Télévision Suisse RTS découverte. La saison 1 est en ligne depuis octobre 2015 sur la plateforme de Francetv Éducation: http:// education.francetv.fr/atome-hotel/.

13 Le projet a impliqué une soixantaine de personnes: trois médiateurs scientifiques, une chargée de communication et cinq stagiaires pour la réalisation des contenus (recherche des sujets à tourner, entretiens avec les chercheurs, recherche bibliographique, événementiels, scénarios, voix off, animations), auxquels s'ajoutent bien sûr, tous les chercheurs interviewés sans lesquels ce projet n'aurait pu se réaliser. L'équipe de réalisation documentaire a été complémentaire : deux producteurs, une chargée de projet multimédia, deux graphistes, un développeur web, un monteur, un réalisateur et son équipe technique (ingénieur du son, chef opérateur), auxquels il faut ajouter un animateur-web 3D, un musicien et les comédiens pour les voix off. Enfin, des partenariats étroits ont permis de finaliser et de créer un contenu riche et diversifié (réseau Canopée, organismes de recherche, enseignants du secondaire).

14 Le travail collaboratif entre deux univers aussi éloignés que l'administration de l'université de Montpellier et la société de production a été aussi enrichissant que porteur de multiples ajustements à réaliser. Dans ce sens, les difficultés rencontrées ont majoritairement été d'ordre temporel :

15 - le plan de financement d'un montant de $316000 €$ a pris du temps à se constituer en sollicitant des partenaires publics tels que la Région Languedoc-Roussillon, le réseau Canopée ou le CNC ;

16 - les engagements avec les diffuseurs (Francetv Éducation, RTS découverte en Suisse) ont également nécessité des délais importants avant de pouvoir être formalisés ;

17 - le temps nécessaire au tournage d'un documentaire était conséquent (environ 7 jours/ projet pour les médiateurs scientifiques) et l'étape de validation du contenu par les chercheurs a été longue.

\section{Atome Hôtel, lauréat d'une bourse de coproduction audiovisuelle et multimédia}

18 En février 2011, dans le contexte de l'opération Investissements d'Avenir voulue par l'État, un consortium piloté par Universcience a déposé le projet ESTIM-Numérique auprès de l'Agence Nationale de la Rénovation Urbaine (ANRU) en réponse à un appel à projets intitulé "Egalité des chances et promotion de la culture scientifique".

Le projet ESTIM-Numérique pour "Égalité d'accès aux Sciences, aux Technologies, à l'Innovation et au Multimédia" a été retenu par l'ANRU en juillet 2011. L'un de ses volets consistait à augmenter et à mutualiser les productions de contenus numériques de culture scientifique, technique et industrielle. Les bourses de coproductions concernaient des œuvres audiovisuelles et multimédia destinées à diffuser la culture scientifique et technique. Tous les types de publics étaient visés, mais une attention particulière a été portée aux projets s'adressant de façon prioritaire aux jeunes de la tranche 12-25 ans peu intéressés au départ aux questions de science.

20 Comme le rappelle Françoise Augier, responsable de la production audiovisuelle à Universcience, "il était ambitieux de vouloir s'attaquer au tableau périodique des éléments de 
Mendeleiev pour en faire un objet attractif pour tous les publics. Force est de constater que l'objectif est atteint. Ludique et pédagogique, Atome Hôtel propose une diversité d'entrées qui le destine au public le plus large. Documentaires, reportages, interviews, archives, cette richesse de l'offre, sur des tons très différents, aiguise la curiosité. On s'y plonge avec facilité, stimulés par une interface claire, une navigation fluide, et un graphisme séduisant, sans jamais se perdre. Le principe du web-doc est ici parfaitement utilisé, réunissant avec cohérence une grande variété de documents et d'approches. Par là-même, Atome Hôtel est une réalisation qui répond aux objectifs fixés par Estim. Restent aux acteurs de la culture scientifique et industriels à s'en emparer pour le diffuser largement auprès de tous les publics".

21 C'est dans ce contexte que le projet Atome Hôtel a été sélectionné et a pu ainsi bénéficier d'une bourse de coproduction ESTIM-Numérique dans le cadre du programme des Investissements d'Avenir. Cette aide financière a constitué un tremplin déterminant pour sa production et sa réalisation.
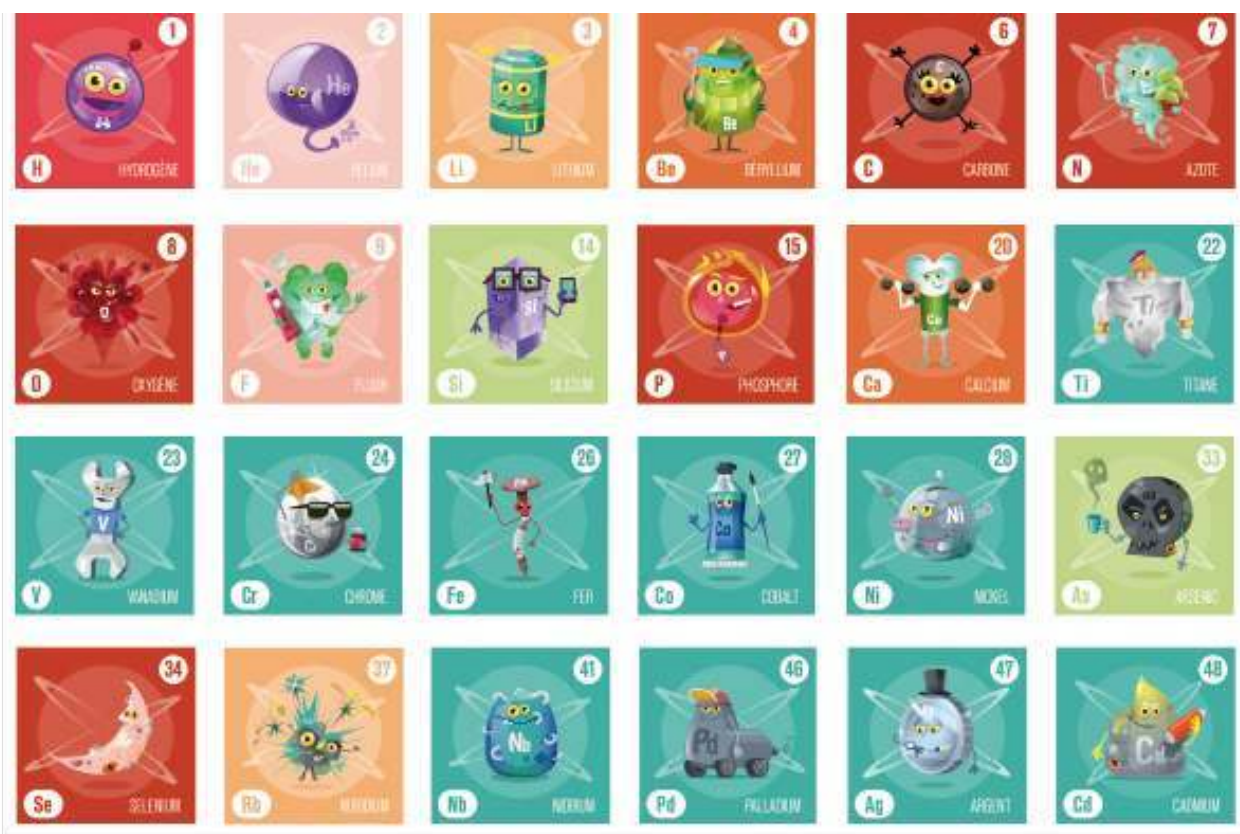

(C) Loguy-Bot 42

\section{Le web-documentaire comme support pédagogique}

La diffusion de la culture scientifique est l'une des missions des universités. Pour Muriel Guedj, vice-présidente à l'université de Montpellier, déléguée à la diffusion de la culture scientifique et à la valorisation des patrimoines historiques "l'objectif est de diminuer les distances géographiques et sociales entre le savoir qui s'exerce dans nos laboratoires et le grand public". Dans cette perspective "Atome hôtel est un programme de culture scientifique qui répond à une mission importante de l'université: celle de diffuser les connaissances scientifiques dans la société. Ancré sur les techniques du numérique, ce web documentaire s'adresse à tous. Accessible, ludique et attractif tout en étant particulièrement rigoureux d'un point de vue scientifique, il constitue un outil solide qui en "mettant les sciences en culture" permet à chacun, de rencontrer et d'interagir avec une science innovante et des acteurs engagés dans des pratiques et des savoirs implantés au coeur de la société". 

axes prioritaires de la stratégie numérique du ministère de l'Éducation nationale, de l'Enseignement supérieur et de la Recherche. La diffusion des usages du numérique dans l'enseignement constitue en effet un puissant levier de modernisation, d'innovation pédagogique et de démocratisation du système scolaire. Atome Hôtel illustre une approche du numérique qui permet de montrer la science de manière innovante et vivante.

Le web-documentaire est une narration interactive multimédia dont la diffusion se fait par le Web. C'est un genre hybride, croisement entre cinéma documentaire et site Internet, qui mêle dans un dosage subtil des séquences filmées, textes, documents photographiques, séquences de jeu, laissant à l'internaute le choix de son parcours. Ce mode d'expression connaît un véritable essor et s'inscrit dans le courant des nouvelles écritures (blog, web fiction, serious game...) qui font leur entrée dans l'enseignement. Interactivité et transmedia s'invitent désormais dans le monde éducatif et celui de la médiation des sciences.

Tournage en septembre 2014 pour la production d'Atome Hôtel à Långban lors du road-trip en Suède avec Jörgen Langhof, responsable des collections minéralogiques du muséum d'Histoire naturelle de Stockholm.

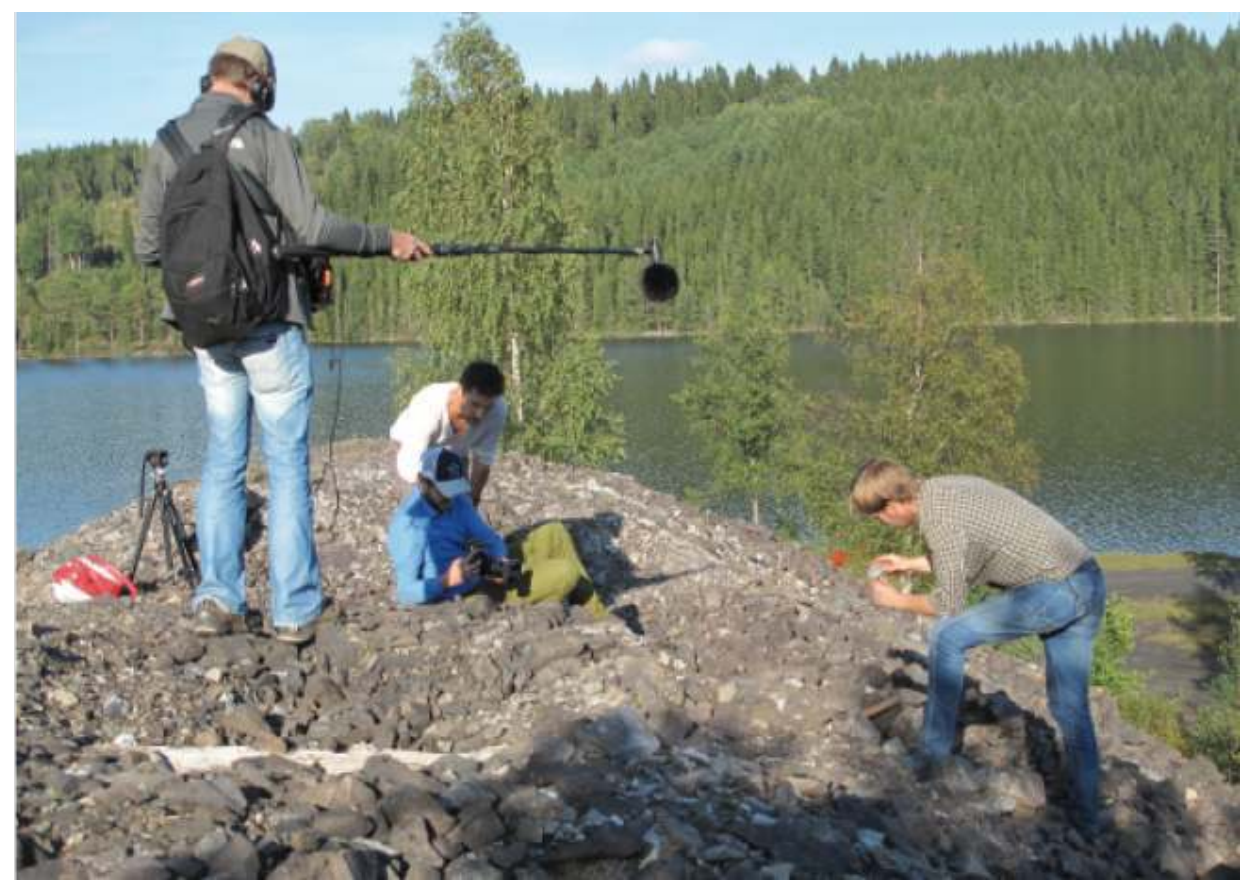

(c) T. Brassac

\section{Interfaces entre réel et virtuel : perspectives et questionnements}

Suite à la mise en ligne de la première phase du projet, se pose désormais la question de son appropriation par les publics. Dans un premier temps, les communautés éducatives sont en première ligne et nous comptons nous donner les moyens de collaborer (formations, rencontres, co-production) avec les enseignants et les élèves. Il est à noter 
que l'étude du tableau périodique des éléments peut concerner les enseignants de multiples horizons et permettre la réalisation de projets transdisciplinaires : littérature, histoire, économie, cinéma, arts plastiques, théâtre... Il est facilement envisageable que des classes (ou des chercheurs) puissent décider d'adopter des atomes et contribuer ainsi à la production de contenus. La plateforme numérique Atome Hôtel pourra ainsi servir de laboratoire d'expérimentations en médiation scientifique numérique; l'hôtel est désormais ouvert et les chambres sont à louer...

La première version du site ne permet qu'une interactivité limitée avec les internautes (quizz, vote pour son élément préféré, réseaux sociaux). Nous réfléchissons à de nouvelles possibilités de collaboration sous la forme d'upload de textes, photographies ou vidéos.

Des études, en partenariat avec l'Éducation Nationale sur l'utilisation dans et hors temps scolaires vont être mises en place. Des outils de médiation ont également été conçus pendant toute la durée du projet pour la promotion d' Atome Hôtel en "face public" : une exposition illustrée sur le concept d'atomes, un atelier scientifique "lumière et atomes", un meuble à "objets atomiques", une bibliothèque atomique, une collection encadrée de tableaux périodiques esthétiques, un jeu des 7 familles, un jeu de plateau atomique.

Il nous semble important que les dimensions réelle et virtuelle du projet puissent se nourrir l'une de l'autre; les rencontres réelles sont souvent propices à la mise en place de collaborations, la réalisation d'interviews ou le partage d'informations; le webdocumentaire et les réseaux sociaux contribuent à construire une communauté numérique qui permet de nouveaux types d'interaction et l'atteinte de nouveaux publics (bloggeurs, anonymes curieux de sciences).

29 La mise en ligne du web-documentaire marque finalement le début d'une nouvelle phase $\mathrm{du}$ projet: publics et futurs collaborateurs peuvent (enfin) découvrir cet univers et ses contenus. Nous sommes prêts à imaginer ensemble la suite de l'aventure numérique.

\section{Le témoignage d'un chercheur}

"Atome Hôtel est un très beau projet avec un point d'entrée original : le tableau périodique des éléments devient ici cabinet de curiosités qui invite à la visite, à la découverte. J'hésitais sur l'atome à adopter tant chacun a son importance en astrophysique. Notre départ imminent pour une semaine à l'Observatoire de Haute-Provence avec les étudiants du master 2 Cosmos Champs et Particules nous a donné l'idée de proposer un tournage sur place. L'hydrogène est rapidement devenu l'atome central de cette aventure. La durée très courte du web documentaire et le public visé rendaient l'exercice difficile. Le scientifique et l'enseignant que je suis se devait pourtant de conserver rigueur et précision au propos. L'interaction durant 2 jours avec l'équipe de tournage a été passionnante, et riche d'échange. J'en garde un très bon souvenir malgré la fatigue du travail de nuit suivi des interviews de jour, la course après la lumière du couchant, avec des séquences qui ne pouvaient être tournées qu'une fois...

Le résultat est très beau et il a une résonance particulière, du fait de l'implication d'étudiants en stage d'initiation à la recherche. C'est une magnifique illustration de ce que nous faisons avec eux et une merveilleuse occasion de promouvoir notre science et les études à l'université. L'une des grandes richesses d'Atome Hôtel est de montrer les chercheurs en action, avec leur enthousiasme et leur envie de partager les mondes qu'ils découvrent. Cela devrait contribuer à rapprocher science et citoyens".

Bertrand Plez, 
chercheur au laboratoire Univers et Particules de Montpellier,enseignant à la faculté des sciences de l'université de Montpellier

\section{Le témoignage des professionnels de l'image}

"Notre ambition, en rejoignant le projet Atome Hôtel, était bien de permettre à cette initiative originale de vulgarisation scientifique, d'être déclinée sous la forme d'un webdocumentaire ambitieux et innovant tant dans son propos (de nombreux films et enquêtes, tournés dans plusieurs pays) que dans son écriture et sa navigation. Ce projet nous a semblé tout à fait pertinent. Immédiatement, nous avons été séduits par une approche de l'atome qui pouvait devenir une source d'inspiration inépuisable, au croisement des approches scientifiques, artistiques et numériques. Atome Hôtel a retenu l'intérêt d'un grand nombre de laboratoires de renom. Il s'appuie donc sur un ensemble de compétences scientifiques hautement qualifiées. Nous avons accompagné chaque étape du projet avec des compétences adaptées à chacune d'entre elles. Nous avons proposé et intégré à l'équipe un designer qui a su apporter une approche graphique originale.

Le but du travail a été d'accompagner le développement des scénarios et la "bible interactive" ainsi que l'approche graphique et technique, et bien sûr d'assurer les tournages des 30 films courts-documentaires".

Pages \& Images Productions, producteur délégué

"Venant de filières humaines et sociales, mettre en scène un web documentaire au label scientifique avec un ceil profane, se révélait être un véritable défi, mais aussi un jeu... pour rendre attrayant ce qui était rédhibitoire dans ces salles de classes de chimie et physique au collège ! L'ambition pour ces épisodes était de faire oublier que l'on est dans un documentaire scientifique éducatif: je viens d'une culture cinématographique que j'essaie de transposer dans le webdoc. Le but est d'offrir de l'élégance par le cadre, la musique, la lumière, la voix off, qui accompagnent et illustrent une rigueur scientifique. Pour cela Atome Hôtel a bénéficié de formidables techniciens qui ont pris part et corps à l'aventure pour délivrer ensemble une partition de belle tenue".

Baptiste Rouveure, réalisateur

www.periodicvideos.com (periodic table of videos, Nottingham University)

http//dublin.sciencegallery.com/elements (exposition art \& science ELEMENTS: the beauty of chemistry proposée par la Science Gallery de Dublin

www.94elements.com (projet cinématographique)

www.chem-toddler.com (site Serbe d'expériences de chimie,cliquer sur "all experiments")

www.periodictable.co.uk (dispositifs interactifs de tableaux périodiques)

\section{BIBLIOGRAPHIE}

Aldersey-Williams, H. Periodic tales: the curious lives of the elements. Édition Penguin Books, 2011. 
Emsley,J. Nature's building blocks : an A-Z guide to the elements. Édition Oxford University Press, 2011.

Fernandez, B. De l'atome au noyau : une approche historique de la physique atomique et de la physique nucléaire. Édition Ellypses, 2006.

Gordin, M.-D. A well ordered thing: Dmitrii Mendeleev and the shadow of the periodic table. Édition Basic Books, 2004.

Gray, T. Atomes : une exploration visuelle de tous les atomes connus dans l'Univers. Édition Place des victoires, 2010.

Gray, T. Molécules : l'architecture du quotidien et de l'infini. Édition Place des victoires, 2014.

Sacks, O. Oncle Tungstène. Édition Seuil, 2003.

Scerri, É. Le tableau périodique : son histoire et sa signification. Édition Oxford Publishing Limited, 2006.

Hors série « Atomique », revue Drosophile

\section{RÉSUMÉS}

Rendre attractif et ludique, le tableau périodique des éléments, cet icône de la chimie : tel est l'objectif que s'est fixé le service de culture scientifique de l'université de Montpellier. Les initiateurs de l'opération présentent les différentes étapes de ce projet qui a finalement pris la forme d'un web-documentaire, fruit d'une collaboration étroite entre des scientifiques et une équipe créative spécialisée dans le multimédia.

\section{AUTEURS}

\section{THIERRY BRASSAC}

responsable du service de culture scientifique de l'université de Montpellier thierry.brassac@umontpellier.fr

\section{AGNÈS PESENTI}

chargée de communication du service de culture scientifique de l'université de Montpellier agnes.pesenti@umontpellier.fr 